\title{
ZETA FUNCTIONS AND THEIR ASYMPTOTIC EXPANSIONS FOR COMPACT LOCALLY SYMMETRIC SPACES OF NEGATIVE CURVATURE
}

\author{
BY ROBERT S. CAHN AND JOSEPH A. WOLF
}

Communicated by S. S. Chern, July 3, 1975

Let $G$ be a noncompact, connected, semisimple Lie group with maximal compact subgroup $K$. Let $\Gamma$ be a discrete, cocompact subgroup of $G$ with no nontrivial elements of finite order and denote by $M$ the space $\Gamma \backslash G / K$. $M$ will be a Riemannian manifold with metric arising from the Cartan-Killing form of the Lie algebra of $G$. The Laplacian of $M$ will have eigenvalues $0=\lambda_{0} \leqslant \lambda_{1} \leqslant \lambda_{2} \leqslant \cdots$ Let $\zeta_{M}(t)=\Sigma_{0}^{\infty} e^{-\lambda_{n} t}$. It is standard that

$$
\zeta_{M}(t) \cong(4 \pi t)^{-\operatorname{dim}(M) / 2}\left(a_{0}+a_{1} t+\cdots+a_{n} t^{n}+O\left(t^{n+1}\right)\right), \quad t \downarrow 0 .
$$

Let $M^{\prime}=G^{\prime} / K$ be the compact dual of $G / K$. Then

$$
\zeta_{M^{\prime}}(t) \cong(4 \pi t)^{-\operatorname{dim}(M) / 2}\left(a_{0}^{\prime}+a_{1}^{\prime} t+\cdots+a_{n}^{\prime} t^{n}+O\left(t^{n+1}\right)\right), \quad t \downarrow 0
$$

and the coefficients $a_{n}^{\prime}$ have been computed (see [1] and [2]).

THEOREM. $a_{n}=(-1)^{n}\left(\operatorname{Vol}(M) / \operatorname{Vol}\left(M^{\prime}\right)\right) a_{n}^{\prime}$.

"Nolan Wallach informs us that Mr. Miatello has proved this result for symmetric spaces of rank 1 using different methods."

\section{BIBLIOGRAPHY}

1. R. Cahn and J. Wolf, Zeta functions and their asymptotic expansions for compact symmetric spaces of rank one, Comment. Math. Helv. (to appear).

2. - Zeta functions and their asymptotic expansions for compact symmetric spaces of rank $n$ (in preparation).

DEPARTMENT OF MATHEMATICS, UNIVERSITY OF MIAMI, CORAL GABLES, FLORIDA 33124

DEPARTMENT OF MATHEMATICS, UNIVERSITY OF CALIFORNIA, BERKELEY, CALIFORNIA 94720

AMS (MOS) subject classifications (1970). Primary 35P20, 43 A85. 